Correction

\title{
Correction: Molines, J., et al. Influence of Parapets on Wave Overtopping on Mound Breakwaters with Crown Walls. Sustainability 2019, 11, 7109
}

\author{
Jorge Molines ${ }^{1, *(1)}$, Arnau Bayon ${ }^{2}\left(\mathbb{D}\right.$, M. Esther Gómez-Martín ${ }^{1}\left[\right.$ and Josep R. Medina ${ }^{1}(\mathbb{C}$ \\ 1 Department of Transportation, Universitat Politècnica de València, Camino de Vera s/n, \\ 46022 Valencia, Spain; mgomar00@upv.es (M.E.G.-M.); jrmedina@upv.es (J.R.M.) \\ 2 Department of Hydraulic Engineering and Environment, Universitat Politècnica de València, Camino de \\ Vera s/n, 46022 Valencia, Spain; arbabar@upv.es \\ * Correspondence: jormollo@upv.es
}

Received: 12 May 2020; Accepted: 13 May 2020; Published: 14 May 2020

The authors would like to make the following corrections about the published paper [1]. The changes are as follows:

(1) Replacing the Equation (3):

$$
\frac{\partial \overline{\mathrm{u}}}{\partial \mathrm{t}}+\overline{\mathrm{u}} \cdot \nabla \overline{\mathrm{u}}=-\frac{1}{\rho} \nabla \mathrm{p}+v \nabla^{2} \overline{\mathrm{u}}+\overline{\mathrm{f}_{\mathrm{b}}}+\mathrm{I}
$$

with

$$
\frac{\partial}{\partial \mathrm{t}}(\rho \overline{\mathrm{u}})+\nabla \cdot\left(\frac{1}{\mathrm{n}} \rho \overline{\mathrm{u}} \times \overline{\mathrm{u}}\right)=-\mathrm{n} \nabla \mathrm{p}+\nabla(u \nabla \cdot \overline{\mathrm{u}})+\mathrm{n} \rho \overline{\mathrm{f}_{\mathrm{b}}}-\mathrm{I},
$$

(2) Replacing the Equation (4):

$$
\frac{\partial \overline{\mathrm{u}}}{\partial \mathrm{t}}+\overline{\mathrm{u}} \cdot \nabla \overline{\mathrm{u}}=-\frac{1}{\rho} \nabla \mathrm{p}+v \nabla^{2} \overline{\mathrm{u}}+\overline{\mathrm{f}_{\mathrm{b}}}+\mathrm{I}
$$

with

$$
\mathrm{I}=A \overline{\mathrm{u}}+\mathrm{B} \overline{\mathrm{u}}|\overline{\mathrm{u}}|+\mathrm{c} \frac{\partial}{\partial \mathrm{t}}(\rho \overline{\mathrm{u}})
$$

(3) Replacing the Equation (5):

$$
\frac{\partial \overline{\mathrm{u}}}{\partial \mathrm{t}}+\overline{\mathrm{u}} \cdot \nabla \overline{\mathrm{u}}=-\frac{1}{\rho} \nabla \mathrm{p}+v \nabla^{2} \overline{\mathrm{u}}+\overline{\mathrm{f}_{\mathrm{b}}}+\mathrm{I}
$$

with

$$
\mathrm{A}=\alpha \frac{(1-\mathrm{n})^{3}}{\mathrm{n}^{2}} \frac{\mu}{\mathrm{D}_{50^{2}}}
$$

(4) Replacing the Equation (7):

$$
\frac{\partial F}{\partial t}+\nabla \cdot(\bar{u} \mathrm{~F})+\nabla \bar{u}_{c} \mathrm{~F}(1-\mathrm{F})=0
$$

with

$$
\frac{\partial F}{\partial t}+\nabla \cdot\left(\frac{1}{\mathrm{n}} \bar{u} \mathrm{~F}\right)+\nabla \cdot\left(\frac{1}{\mathrm{n}} \bar{u}_{c}\right) \mathrm{F}(1-\mathrm{F})=0,
$$


The authors and the Editorial Office would like to apologize for any inconvenience caused to the readers by these changes. The change does not affect the scientific results. The manuscript will be updated and the original will remain online on the article webpage.

\section{References}

1. Molines, J.; Bayon, A.; Gómez-Martín, M.E.; Medina, J.R. Influence of Parapets on Wave Overtopping on Mound Breakwaters with Crown Walls. Sustainability 2019, 11, 7109. [CrossRef] 